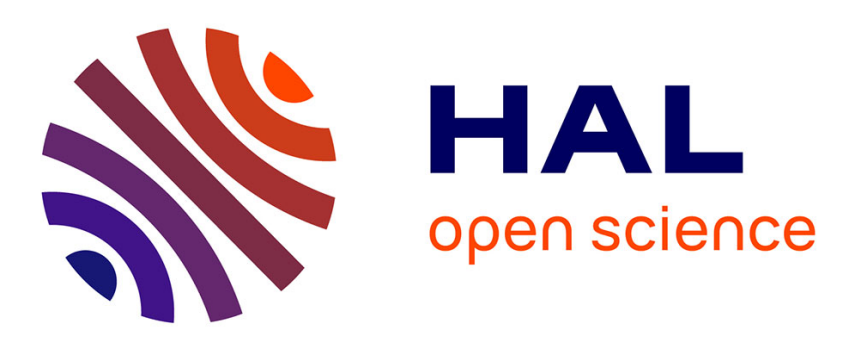

\title{
Proton-proton physics with the ALICE muon spectrometer at the LHC
}

N. Bastid

\section{To cite this version:}

N. Bastid. Proton-proton physics with the ALICE muon spectrometer at the LHC. The 19th International Conference on Ultra-Relativistic Nucleus-Nucleus Collisions, Nov 2006, Shanghai, China. pp.2438-2444, 10.1142/S0218301307008069 . in2p3-00150369

\section{HAL Id: in2p3-00150369 https://hal.in2p3.fr/in2p3-00150369}

Submitted on 30 May 2007

HAL is a multi-disciplinary open access archive for the deposit and dissemination of scientific research documents, whether they are published or not. The documents may come from teaching and research institutions in France or abroad, or from public or private research centers.
L'archive ouverte pluridisciplinaire HAL, est destinée au dépôt et à la diffusion de documents scientifiques de niveau recherche, publiés ou non, émanant des établissements d'enseignement et de recherche français ou étrangers, des laboratoires publics ou privés. 


\title{
PROTON-PROTON PHYSICS WITH THE ALICE MUON SPECTROMETER AT THE LHC
}

\author{
N. BASTID for the ALICE Collaboration \\ LPC Clermont-Ferrand, IN2P3-CNRS and Université Blaise Pascal \\ 63177 Aubière Cedex, France \\ nicole.bastid@clermont.in2p3.fr \\ Received (March 3rd)
}

\begin{abstract}
ALICE, the dedicated heavy ion experiment at the LHC, will also have an important impact on the proton-proton physics program. The physics analyses foreseen with the ALICE muon spectrometer are reviewed. A particular emphasis is placed on heavy flavor measurement.
\end{abstract}

\section{Introduction}

With a nucleon-nucleon center-of-mass energy for $\mathrm{Pb}-\mathrm{Pb}$ collisions that will exceed the one reached at RHIC by about a factor 30, the LHC (Large Hadron Collider) will offer new insights in heavy ion physics. ALICE (A Large Ion Collider Experiment), the only experiment devoted to heavy ion physics at the LHC, will investigate properties of strongly interacting matter at extreme energy densities and temperatures. The successful achievement of the heavy ion program requires also the study of proton-proton, proton-nucleus and light nucleus-nucleus systems. Proton-proton collisions are of great interest since besides providing the necessary baseline for nucleus-nucleus collisions, they allow to test both perturbative and non-perturbative regimes of $\mathrm{QCD}$ in a new kinematic region associated with very low Bjorken- $x$ values. Moreover, the ALICE proton-proton physics program is complementary to the studies performed by the dedicated proton-proton experiments at the LHC.

The LHC is scheduled to start to operate in fall 2007. It will deliver, in nominal conditions, proton beams at $\sqrt{s}=14 \mathrm{TeV}$ several months per year (data taking time $\mathrm{t} \sim 10^{7} \mathrm{~s}$, average ALICE luminosity $\langle\mathfrak{L}\rangle=3.10^{30} \mathrm{~cm}^{-2} \mathrm{~s}^{-1}$ ) and Pb beams at $\sqrt{s_{N N}}=5.5 \mathrm{TeV}$ one month per year $\left(\mathrm{t} \sim 10^{6} \mathrm{~s},\langle\mathfrak{L}\rangle=5.10^{26} \mathrm{~cm}^{-2} \mathrm{~s}^{-1}\right)$. It is also foreseen that the LHC provides light ion beams. This will allow the study of both symmetric and asymmetric systems, such as proton-nucleus.

In the following, after a short description of the apparatus, we will discuss selected proton-proton physics topics that will be studied with the ALICE muon spectrometer. The ALICE heavy ion program as well as proton-proton physics with the central barrel were presented at this conference. ${ }^{1}$ 


\section{Overview of the ALICE muon spectrometer}

The ALICE apparatus, under installation, consists of a central barrel $(|\eta|<0.9)$ placed in the L3 magnet, a forward muon spectrometer and other sub-detectors of smaller acceptance. ${ }^{2}$

The main aim of the ALICE muon spectrometer is the study of heavy flavor production (open heavy flavors and quarkonia) in the (di)muon channel. ${ }^{3}$ In addition, the production of weakly interacting probes $\left(Z^{0}\right.$ and $W^{ \pm}$bosons) and low mass resonances $(\rho, \omega, \phi)$ is also investigated.

The main design criteria are driven by the requirements that the detector should operate in the high multiplicity environment of central $\mathrm{Pb}-\mathrm{Pb}$ collisions at $\sqrt{s_{N N}}=$ 5.5 TeV and should reach a mass resolution of $100 \mathrm{MeV} / c^{2}$ in the $\Upsilon$ mass region in order to resolve the $\Upsilon(1 S), \Upsilon(2 S)$ and $\Upsilon(3 S)$ states. The ALICE muon spectrometer covers the polar angular range $171^{\circ}<\theta<178^{\circ}$ which corresponds to a pseudorapidity range $-4.0<\eta<-2.5$. It is composed of a passive front absorber, a small angle absorber, a dipole magnet, five stations of high granularity tracking chambers, a muon filter and two stations of trigger chambers.

\section{Heavy flavor production}

At the LHC, hard processes will contribute significantly to the total cross section. Expected yields and cross sections of $c \bar{c}$ and $b \bar{b}$ pairs, calculated in the framework of perturbative QCD (pQCD) at next-to-leading order (NLO), are reported in Table 1 for the $5 \%$ most central $\mathrm{Pb}-\mathrm{Pb}$ collisions at $\sqrt{s_{N N}}=5.5 \mathrm{TeV}$ and for $\mathrm{p}-\mathrm{p}$ collisions at $\sqrt{s}=14 \mathrm{TeV}^{4,5}$

Table 1. ALICE baseline for heavy quark production cross sections and yields in p-p collisions $(\sqrt{s}=14 \mathrm{TeV})$ and in central $\mathrm{Pb}-\mathrm{Pb}$ collisions $\left(\sqrt{s_{N N}}=5.5\right.$

$\mathrm{TeV}$ ). For $\mathrm{Pb}-\mathrm{Pb}$ collisions, nuclear shadowing is included and a binary scaling is applied.

\begin{tabular}{ccccc}
\hline System & $N_{c \bar{c} / \text { event }}$ & $\sigma_{c \bar{c}}^{N N}(m b)$ & $N_{b \bar{b} / \text { event }}$ & $\sigma_{b \bar{b}}^{N N}(m b)$ \\
\hline $\mathrm{Pb}-\mathrm{Pb}\left(\sqrt{s_{N N}}=5.5 \mathrm{TeV}\right)$ & 115 & 4.32 & 4.56 & 0.18 \\
$\mathrm{p}-\mathrm{p}(\sqrt{s}=14 \mathrm{TeV})$ & 0.16 & 11.2 & 0.0072 & 0.51 \\
\hline
\end{tabular}

Heavy flavors are produced in the early stage of the collision and, due to their long life-time, they allow to probe the dynamics of the hot and dense system created in heavy ion collisions. In this regard, the study of p-p collisions is of crucial interest for the interpretation of results obtained in A-A and p-A collisions in order to unravel the effects induced by the nuclear medium (such as shadowing and quenching effects). In addition, the measurement of charm and beauty cross sections in p-p collisions at $\sqrt{s}=14 \mathrm{TeV}$ should constrain free parameters of NLO pQCD calculations since the theoretical uncertainties on the absolute values are about a factor $2-3 .{ }^{5}$ However, simulation results indicate that an extrapolation, based on 
pQCD calculations, can be used to compare charm and beauty cross sections measured in p-p $(\sqrt{s}=14 \mathrm{TeV})$ and in $\mathrm{Pb}-\mathrm{Pb}\left(\sqrt{s_{N N}}=5.5 \mathrm{TeV}\right)$ collisions since the theoretical uncertainty introduced by the extrapolation is only a few $\% .^{5}$

\subsection{Open beauty production}

In addition to the motivations discussed above, the study of open beauty production in $\mathrm{p}-\mathrm{p}$ collisions is of particular relevance for understanding bottomonium production in $\mathrm{p}-\mathrm{p}, \mathrm{p}-\mathrm{A}$ and A-A collisions. Such measurement provides the most natural normalization for $\Upsilon$ yields because of similar production processes at the partonic level. The measurement of the $B$ hadron cross section is also mandatory to estimate the contribution of secondary $J / \psi$ (from $B$ decays) to the total $J / \psi$ yield.

The possibility to determine the differential $B$ hadron cross section has been first investigated for central $(5 \%) \mathrm{Pb}-\mathrm{Pb}$ collisions at $\sqrt{s_{N N}}=5.5 \mathrm{TeV}$ by means of fast simulations and by using a method developed by the UA1 collaboration in $\mathrm{p}-\overline{\mathrm{p}}$ collisions. ${ }^{6}$ The cross section is determined from the measured single muon transverse momentum $\left(p_{t}\right)$ distribution as well as from the unlike-sign dimuon yields in the low mass region (each muon originates from the same $B$ meson through a $D$ meson decay) and in the high mass region (each muon comes from the direct decay of a $B$ meson). ${ }^{5,7}$ In one data taking period, one expects a large statistics of single muons and unlike-sign dimuons from open beauty over a wide $p_{t}$ and mass range, respectively. ${ }^{7}$ Figure 1 shows that the $B$ hadron cross section can be measured up to about $30 \mathrm{GeV} / c$ and that a remarkable agreement between the different channels is achieved.

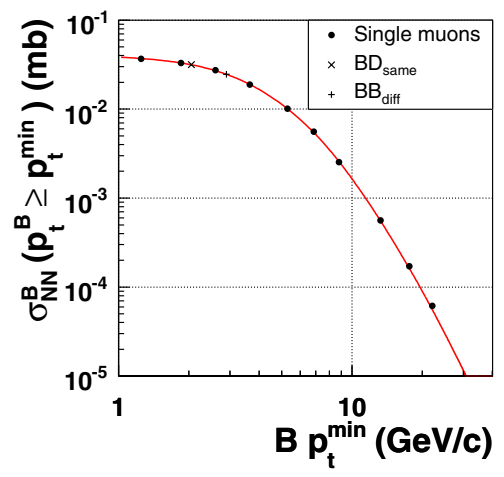

Fig. 1. Differential $B$ hadron inclusive production cross section in central $\mathrm{Pb}-\mathrm{Pb}$ collisions $\left(\sqrt{s_{N N}}\right.$ $=5.5 \mathrm{TeV})$ for single muons, low mass unlike-sign dimuons $\left(B D_{\text {same }}\right)$ and high mass unlike-sign dimuons $\left(B B_{d i f f}\right)$. The input cross section used in the simulation is also displayed (solid curve).

The preliminary statistics estimates for a nominal p-p run at $14 \mathrm{TeV}$ are presented in Table $2 .{ }^{8}$ In the acceptance of the ALICE muon spectrometer, one expects 
a huge statistics of single muons and unlike-sign dimuons from open beauty. We have also estimated the yields for a reduced sample of events, as it could be collected during the first weeks of data taking at $14 \mathrm{TeV}$, assuming an average luminosity of $10^{30} \mathrm{~cm}^{-2} \mathrm{~s}^{-1}$ and an effective time of $7 \cdot 2.10^{5} \mathrm{~s}$ (run-1). This suggests that the measurement of single muon $p_{t}$ distribution can be used to provide a first measurement of the open beauty cross section at forward rapidities in ALICE.

Table 2. Expected yields of single muons and unlike-sign dimuons from open beauty in the ALICE muon spectrometer for p-p collisions at $14 \mathrm{TeV}$. See text for details.

\begin{tabular}{cccc}
\hline$b \rightarrow \mu X$ & $p_{t}(\mathrm{GeV} / c)$ & nominal run & run-1 \\
\hline $1.5-3$ & $8.3 .10^{7}$ & $2.0 .10^{6}$ \\
$3-6$ & $2.6 .10^{7}$ & $6.3 .10^{5}$ \\
& $6-9$ & $2.2 .10^{7}$ & $5.3 .10^{4}$ \\
& $9-20$ & $4.7 .10^{5}$ & $1.1 .10^{4}$ \\
\hline$b \bar{b} \rightarrow \mu^{+} \mu^{-}$ & $\mathrm{M}\left(\mathrm{GeV} / c^{2}\right)$ & nominal run & run-1 \\
\hline & $0.3-5$ & $7.3 .10^{5}$ & $1.8 .10^{4}$ \\
& $5-20$ & $1.2 .10^{5}$ & $3.0 .10^{3}$ \\
\hline
\end{tabular}

Open beauty cross section can also be measured by means of $J / \psi$ from $B$ hadron decay. In p-p collisions at $14 \mathrm{TeV}$, this contribution represents about $22 \%$ (in $4 \pi$ ) of the total $J / \psi$ yield. Feasibility studies have demonstrated the possibility to isolate these secondary $J / \psi$ in p-p collisions by triggering on three-muon events. ${ }^{5}$ This component can be identified with a signal to background ratio $\mathrm{S} / \mathrm{B}=3$ and $\mathrm{a}$ significance of 80 in a nominal p-p run at $\sqrt{s}=14 \mathrm{TeV}$.

\subsection{Quarkonium production}

The study of quarkonium production should be combined with that of open heavy flavors. The measurement of quarkonia in $p$ - $p$ collisions is of great interest since it is expected to provide information on production mechanisms in a new energy range and it should allow to extract information on parton distribution functions (PDF) at very small Bjorken- $x$ values, down to about $10^{-5}$. It is also mandatory for the determination of the nuclear modification factor in $\mathrm{Pb}-\mathrm{Pb}$ collisions.

Charmonium and bottomonium production in p-p collisions at $\sqrt{s}=14 \mathrm{TeV}$ has been investigated by means of fast simulations. ${ }^{5,9}$ The invariant mass of unlikesign dimuons leads to a direct measurement of quarkonium production. The results, corresponding to a standard p-p run at $\sqrt{s}=14 \mathrm{TeV}$, show that charmonium and bottomonium states (Fig. 2) are well separated. All quarkonium states are identified with a good significance and the corresponding signal to background (S/B) ratios are always greater than one, except for $\psi^{\prime}(\mathrm{S} / \mathrm{B}=0.62)$. The statistics is very large, in particular for $J / \psi\left(\mathrm{S}=2.8 .10^{6}\right)$ and should allow for detailed analyses as a function of rapidity and transverse momentum. In particular, the rapidity 
dependence of the $J / \psi$ yield should be a relevant observable to unravel PDF at very low Bjorken- $x$ values. ${ }^{9}$ The $J / \psi$ polarization is an other promising observable for testing different production mechanisms in p-p collisions. Recent investigations have demonstrated the capabilities of the ALICE muon spectrometer to measure this observable in p-p collisions at $\sqrt{s}=14 \mathrm{TeV}$, even with a limited statistics. ${ }^{10}$

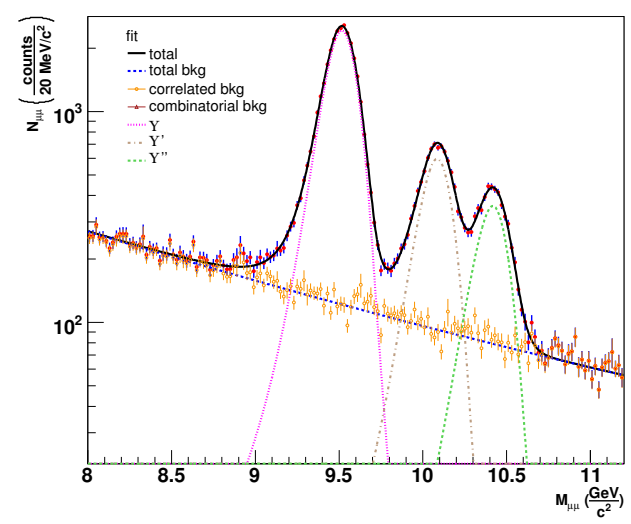

Fig. 2. Invariant mass distribution of unlike-sign dimuons, in the $\Upsilon$ region, in p-p collisions at $\sqrt{s}=14 \mathrm{TeV}$.

\section{Production of electroweak $W^{ \pm}$and $Z^{0}$ bosons}

Massive electroweak $W^{ \pm}$bosons are produced in hard primary collisions. These bosons decay into muons, mainly in the channel $W^{ \pm} \rightarrow \mu^{ \pm} \nu_{\mu}$. In p-p collisions at $\sqrt{s}=14 \mathrm{TeV}$, they will allow to probe $\mathrm{PDF}$ at large $Q^{2}\left(Q^{2} \sim m_{W}\right)$ in the Bjorken-x range from $\sim 10^{-4}$ to $\sim 10^{-3}$.

The dominant process for $W^{+}\left(W^{-}\right)$production is $u \bar{d}(d \bar{u})$ scattering. As protons are made up of $u u d$ valence quarks, $W+$ will in p-p collisions be more abundantly produced than $W^{-}$, leading to a larger yield of $\mu^{+}$than $\mu^{-} \cdot{ }^{11,12}$ This asymmetry is more pronounced at the large rapidities, covered by the ALICE muon spectrometer. As can be seen in Fig. $3, W^{ \pm}$production exhibits a maximum around $40 \mathrm{GeV} / c$ and dominates the high range of the single muon $p_{t}$ distribution. In a nominal p-p run at $14 \mathrm{TeV}$, more than $5.10^{5} W^{ \pm}$bosons decaying into muons will be produced. This corresponds to about $8.6 .10^{4}$ muons in the acceptance of the ALICE muon spectrometer. It has also been shown that the $p_{t}$ dependence of the $N\left(\mu^{+}\right) / N\left(\mu^{-}\right)$ratio is a promising observable for $W^{ \pm}$production. ${ }^{11,12}$

Preliminary studies reveal that $Z^{0}$ bosons also can be reconstructed in the ALICE muon spectrometer by means of the invariant mass of unlike-sign muon pairs. ${ }^{12}$ The estimated yield for one year of data taking is about 2500 . 


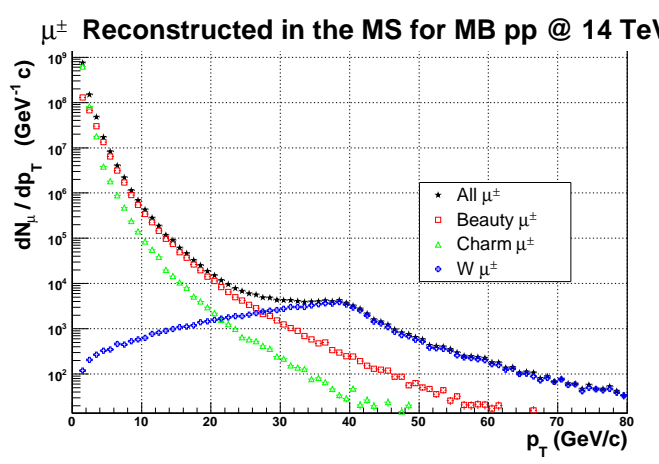

Fig. 3. $p_{t}$ distribution of single muons reconstructed in the acceptance of the ALICE muon spectrometer (stars), in p-p collisions at $\sqrt{s}=14 \mathrm{TeV}$. Squares, triangles and crosses correspond to muons from beauty, charm and $W^{ \pm}$decays, respectively.

\section{Summary}

An ambitious proton-proton physics program, through a large variety of physics channels, will be carried out with the ALICE muon spectrometer at the LHC. It will bring significant insights in a new energy domain. The detector, which is in its final installation phase, will be ready for operation with first proton beams scheduled for November 2007. Intensive preparation for data taking is underway. Full simulations with high statistics data produced via the "Physics Data Challenge" are in progress.

\section{References}

1. F. Antinori for the ALICE Collaboration, proceedings of Quark Matter 2006 conference, nucl-ex/0702013, to be published in J. Phys. G; G. Martinez for the ALICE Collaboration, proceedings of Quark Matter 2006 conference, nucl-ex/0702053, to be published in J. Phys. G; T. K. Nayak for the ALICE Collaboration, proceedings of Quark Matter 2006 conference, nucl-ex/0701067, to be published in J. Phys. G.

2. http://aliceinfo.cern.ch/.

3. F. Carminati et al., ALICE Collaboration, J. Phys. G: Nucl. Part. Phys. 30 (2004) 1517; Alice Collaboration, CERN/LHCC 96-21 (1996); C. Finck for the ALICE Collaboration, J. Phys.: Conf. Series 50 (2006) 397.

4. M. Mangano, P. Nason and G. Ridolfi, Nucl. Phys. B373 (1992) 295.

5. B. Alessandro et al., ALICE Collaboration, J. Phys. G: Nucl. Part. Phys. 32 (2006) 1295.

6. C. Albajar et al., Phys. Lett. B213 (1988) 405; C. Albajar et al., Phys. Lett. B256 (1991) 121.

7. R. Guernane et al., ALICE-INT-2005-018 (2005).

8. N. Bastid, ECT International Workshop on Heavy Flavor Physics in Heavy Ion Collisions at the LHC (2006).

9. D. Stocco et al., ALICE-INT-2006-029 (2006).

10. R. Arnaldi and E. Scomparin, http://www-dapnia.cea.fr/Sphn/Alice/DiMuonNet/.

11. Z. Conesa del Valle et al., ALICE-INT-2006-021 (2006).

12. Z. Conesa del Valle for the ALICE Collaboration, Eur. Phys. J. C49 (2007) 149. 